\title{
Resonance of Superconducting Microstrip Antenna with Aperture in the Ground Plane
}

\author{
Siham Benkouda ${ }^{1}$, Tarek Fortaki ${ }^{2 *}$, Mounir Amir ${ }^{2}$, and Abdelmadjid Benghalia ${ }^{1}$ \\ ${ }^{1}$ Electronics Department, University of Constantine 1, Constantine, Algeria \\ ${ }^{2}$ Electronics Department, University of Batna, Batna, Algeria \\ *corresponding author, E-mail: $t$ fortakie yahoo. fr
}

\begin{abstract}
This paper presents a rigorous full-wave analysis of a high $T_{\mathrm{c}}$ superconducting rectangular microstrip antenna with a rectangular aperture in the ground plane. To include the effect of the superconductivity of the microstrip patch in the full-wave analysis, a complex surface impedance is considered. The proposed approach is validated by comparing the computed results with previously published data. Results showing the effect of the aperture on the resonance of the superconducting microstrip antenna are given.
\end{abstract}

\section{Introduction}

Rectangular microstrip patches can find application in microwave integrated circuit as planar resonators. Also, they can be used as resonant antennas. When a microstrip patch resonator acts as an antenna, the microstrip patch can be fed through an aperture cut into a microstrip line ground plane [1]. This feeding configuration has been found very advantageous for several reasons [2,3]. For instance, it makes it possible to use a high dielectric-constant substrate for the feeding network and a low dielectric-constant substrate for the antenna element, which yields optimal performance for both the feeding network and antenna element. Also, the radiation mechanism from the feeding network cannot interfere with the main radiation pattern generated by the antenna since the ground plane separates the two radiation mechanisms [2]. In addition, the presence of aperture on the ground plane adds new design parameters that can be used to tune the antenna impedance and resonance frequency, without modifying the radiating patch itself [3]. A rectangular coupling aperture introduces two physical parameters: its length and width. Since the presence of apertures in the ground planes of microstrip patches affects the resonant properties of microstrip patch antennas and microstrip patch circuit components, the theoretical model developed for the analysis of microstrip patches should be able to account for the effect of possible apertures existing in the ground plane of the antennas.

Setting aside the topic of microstrip patches over ground planes with apertures, recently there is some interest in the use of superconducting materials in microwave integrated circuits, which is due to their main characteristics, such as: very small losses, which means low-attenuation and lownoise, very small dispersion up to frequencies of several tens of $\mathrm{GHz}$, smaller devices due to the lower losses [4,5], which leads to larger integration density and reduction in the time of propagation of the signals in the circuits.

In this paper, we present a rigorous full-wave analysis of a high $T_{\mathrm{c}}$ superconducting rectangular microstrip antenna with a rectangular aperture in the ground plane. This paper is organized as follows. In section 2, we give some details about the theoretical formulation of the problem. In section 3 , to validate the proposed approach, our numerical results are compared with previously published data. Also in this section, numerical results are presented for the effect of the aperture on the resonance of the high $T_{\mathrm{c}}$ superconducting rectangular microstrip antenna. Finally, concluding remarks are summarized in section 4.

\section{Theory}

The problem to be solved is illustrated in Figure 1. We have a high $T_{\mathrm{c}}$ superconducting rectangular microstrip patch of thickness $e_{1}$ over a ground plane with a rectangular aperture. The superconducting patch is characterized by a critical temperature $T_{c}$, a zero-temperature penetration depth $\lambda_{0}$ and a normal state conductivity $\sigma_{n}$. Let $\mathbf{J}_{0}(x, y)$ be the surface current density on the ground plane with rectangular aperture and let $\mathbf{J}(x, y)$ be the surface current density on the superconducting rectangular patch. Also, let $\mathbf{E}(x, y, 0)$ and $\mathbf{E}(x, y, d)$ are the values of the transverse electric field at the plane of the aperture and at the plane of the superconducting patch, respectively. Following a mathematical reasoning similar to that shown in [3], we can obtain a relation among $\mathbf{J}_{0}(x, y), \mathbf{J}(x, y), \mathbf{E}(x, y, 0)$ and $\mathbf{E}(x, y, d)$ in the vector Fourier transform domain given by

$$
\begin{aligned}
& \mathbf{e}\left(\mathbf{k}_{s}, d\right)=\overline{\mathbf{G}}\left(\mathbf{k}_{s}\right) \cdot \mathbf{j}\left(\mathbf{k}_{s}\right)+\overline{\boldsymbol{\Gamma}}\left(\mathbf{k}_{s}\right) \cdot \mathbf{e}\left(\mathbf{k}_{s}, 0\right),(1) \\
& \mathbf{j}_{0}\left(\mathbf{k}_{s}\right)=-\overline{\boldsymbol{\Gamma}}\left(\mathbf{k}_{s}\right) \cdot \mathbf{j}\left(\mathbf{k}_{s}\right)+\overline{\mathbf{Y}}\left(\mathbf{k}_{s}\right) \cdot \mathbf{e}\left(\mathbf{k}_{s}, 0\right),
\end{aligned}
$$

where the $2 \times 2$ diagonal matrices $\overline{\mathbf{G}}\left(\mathbf{k}_{s}\right), \overline{\boldsymbol{\Gamma}}\left(\mathbf{k}_{s}\right)$ and $\overline{\mathbf{Y}}\left(\mathbf{k}_{s}\right)$ stand for a set of dyadic Green's function, in the vector Fourier transform domain. It is to be noted that $\overline{\mathbf{G}}\left(\mathbf{k}_{s}\right)$ is related to the patch current and $\overline{\mathbf{Y}}\left(\mathbf{k}_{s}\right)$ is related to the aperture field. The matrix $\overline{\mathbf{Y}}\left(\mathbf{k}_{s}\right)$ represents the 


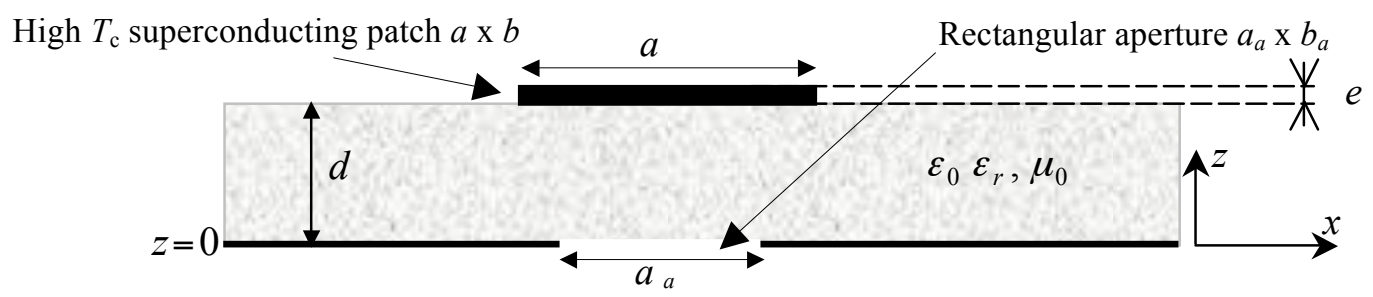

Figure 1: Geometrical structure of a high $T_{\mathrm{c}}$ superconducting rectangular microstrip antenna with a rectangular aperture in the ground plane.

mutual coupling between the patch current and aperture field. In (1) and (2) the unknowns are $\mathbf{j}\left(\mathbf{k}_{s}\right)$ and $\mathbf{e}\left(\mathbf{k}_{s}, 0\right)$. Another possible choice in the analysis of microstrip patches over ground planes with apertures is to consider $\mathbf{j}_{0}\left(\mathbf{k}_{s}\right)$ as unknown instead of $\mathbf{e}\left(\mathbf{k}_{s}, 0\right)$. It is anticipated, however, that a very large number of terms of basis functions would be needed for the expansion of the current $\mathbf{J}_{0}(x, y)$ on the ground plane with aperture because of the wide conductor area. Hence, it is better to apply the Galerkin procedure to the unknown $\mathbf{E}(x, y, 0)$ field at the aperture. The same reasoning has been applied previously in the analysis of finlines [6].

Considering the superconducting effects, we need simply to modify equation (1) by replacing $\overline{\mathbf{G}}\left(\mathbf{k}_{s}\right)$ with $\overline{\mathbf{G}}\left(\mathbf{k}_{s}\right)-Z_{s} \cdot \mathbf{I}$, where $\mathbf{I}$ is the $2 \times 2$ unit matrix and $Z_{s}$ is the complex surface impedance of the superconducting rectangular patch. When the thickness of the superconducting patch is less than three times the zerotemperature penetration depth $\left(\lambda_{0}\right), Z_{s}$ can be expressed as follows:

$$
Z_{s}=\frac{1}{e \sigma},
$$

where $\sigma$ is the complex conductivity of the superconducting film. It is determined by using London's equation and the Gorter-Casimir two-fluid model as $[7,8]$

$$
\sigma=\sigma_{n}\left(\frac{T}{T_{c}}\right)^{4}-\mathrm{i} \frac{1-\left(\frac{T}{T_{c}}\right)^{4}}{\omega \mu_{0} \lambda_{0}^{2}},
$$

where $T$ is the temperature, $T_{c}$ is the transition temperature, $\sigma_{n}$ is the normal state conductivity at $T=T_{c}$ and $\omega$ is the angular frequency. Now, that we have included the effect of the superconductivity of the rectangular patch in the Green's function formulation, the well-known Galerkin procedure of the moment method can be easily applied to obtain the resonant characteristics of the resonant modes of the high $T_{c}$ superconducting rectangular microstrip antenna with a rectangular aperture in the ground plane

\section{Numerical results and discussion}

\subsection{Convergence and comparison of numerical results}

The proper choice of basis functions is the key factor for efficiently calculating accurate results. The basis functions considered here for approximating the unknown patch current are formed by the set of TM modes of a rectangular cavity with magnetic side walls. In [3], Fortaki and Benghalia showed that these basis functions are very appropriate for the vector Fourier transform domain analysis of rectangular microstrip patches over ground planes with rectangular apertures for three reasons: they ensure a quick convergence of the Galerkin's method with respect to the number of basis functions, they lead to vector Fourier transform domain infinite integrals, which are amenable to asymptotic analytical integration techniques and their vector Fourier transforms can be obtained in closed form. Fortunately, all these advantages are kept when the aforementioned basis functions are used in the vector Fourier transform domain analysis of rectangular microstrip antennas with rectangular apertures in the ground plane. Also, the same basis functions are used for approximating the magnetic current density on the aperture in accordance with the concept of complementary electromagnetic structures [3]. Through numerical convergence checks, it is found that for small aperture, only a few number of basis functions suffices to obtain good convergence, while for wide aperture, especially when the size of the aperture is comparable to that of the patch, additional basis functions should also be included in both the approximation of the current density on the rectangular patch and the transverse electric field at the rectangular aperture.

In order to confirm the computation accuracy, our results are compared with the theoretical results of Richard et al. [7] and Silva et al. [8] when there is no aperture in the ground plane. The patch is fabricated with a YBCO superconducting thin film with parameters $\sigma_{n}=10^{6} \mathrm{~S} / \mathrm{M}, T_{c}=$ $89 \mathrm{~K}, \lambda_{0}=140 \mathrm{~nm}$ and $e=350 \mathrm{~nm}$. The patch size is $a \times b=$ $1630 \mu \mathrm{m} \times 935 \mu \mathrm{m}$ and the substrate has a thickness of $d=$ $254 \mu \mathrm{m}$. The operating temperature is $T=50 \mathrm{~K}$. Table 1 summarizes our computed resonant frequencies and those of [7] and [8] for three different materials and differences between these three results of less than $0.7 \%$ are obtained. As a consequence, excellent agreement between our results and those of the literature is achieved. 
Table 1: Comparison of our calculated resonant frequencies with previously published data.

\begin{tabular}{cccc}
\hline \multirow{2}{*}{$\begin{array}{c}\text { Relative } \\
\text { permittivity } \\
\left(\varepsilon_{r}\right)\end{array}$} & \multicolumn{3}{c}{ Resonant frequencies $(\mathrm{GHz})$} \\
\cline { 2 - 4 } & $\begin{array}{c}\text { Cavity } \\
\text { model [7] }\end{array}$ & $\begin{array}{c}\text { Full-wave } \\
\text { analysis [8] }\end{array}$ & Our results \\
\hline 11 & 41.638 & 41.041 & 41.585 \\
16 & 35.300 & 34.856 & 34.816 \\
23.81 & 28.937 & 28.671 & 28.764 \\
\hline
\end{tabular}

\subsection{Influence of the thickness of the patch}

The influence of the thickness of the high $T_{c}$ superconducting film on the operating frequency of antennas with and without apertures is investigated in Figure 2. The thickness of the superconducting patch is normalized with respect to the zero-temperature penetration depth. For the case of structures with apertures, the size of the aperture is $a_{a} \times b_{a}=163 \mu \mathrm{m} \times 93.5 \mu \mathrm{m}$. The superconducting film characteristics are: $\sigma_{n}=9.8310^{5} \mathrm{~S} / \mathrm{m}, T_{c}=89 \mathrm{~K}$ and $\lambda_{0}=100 \mathrm{~nm}$. For the microstrip antenna, the following parameters are used: $a=1630 \mu \mathrm{m}, b=935 \mu \mathrm{m}, \varepsilon_{r}=23.81$ and $d=254 \mu \mathrm{m}$. The operating temperature is $T=50 \mathrm{~K}$. It is noticed that the resonant frequencies of the superconducting patches over ground planes without apertures are larger than those obtained with rectangular apertures. This result agrees with that discovered theoretically for perfectly conducting patches [3].

It is also observed that when the thickness of the superconducting film grows, the resonant frequency increases for antennas without apertures as well as for antennas with apertures. Note that the effect of the thickness of the superconducting film is more pronounced for small values of $e \quad\left(e<0.1 \lambda_{0}\right)$. When $e$ exceeds $0.1 \lambda_{0}$, increasing the superconducting film thickness will increase slowly the resonant frequency. Extreme care should be taken when designing an antenna with thin superconducting film; since small uncertainty in $e$ when the patch is fabricated can result in an important shift of the resonant frequency.

\subsection{Influence of the temperature}

Numerical results are obtained for the parameters used in the experiment of Richard et al. [7] with the simple difference that in our considered structure the ground plane has a rectangular aperture of size $a_{a} \times b_{a}=163 \mu \mathrm{m} \times 93.5 \mu \mathrm{m}$. A rectangular patch with a thickness $350 \mathrm{~nm}$, a resonant length $935 \mu \mathrm{m}$ and a width of $1630 \mu \mathrm{m}$ is printed on a lanthanum aluminate substrate of thickness $254 \mu \mathrm{m}$. The patch is fabricated using a YBCO superconducting thin film with a transition temperature $T_{c}=89 \mathrm{~K}$, a zero-temperature penetration depth $\lambda_{0}=140 \mathrm{~nm}$ and a normal state conductivity at the

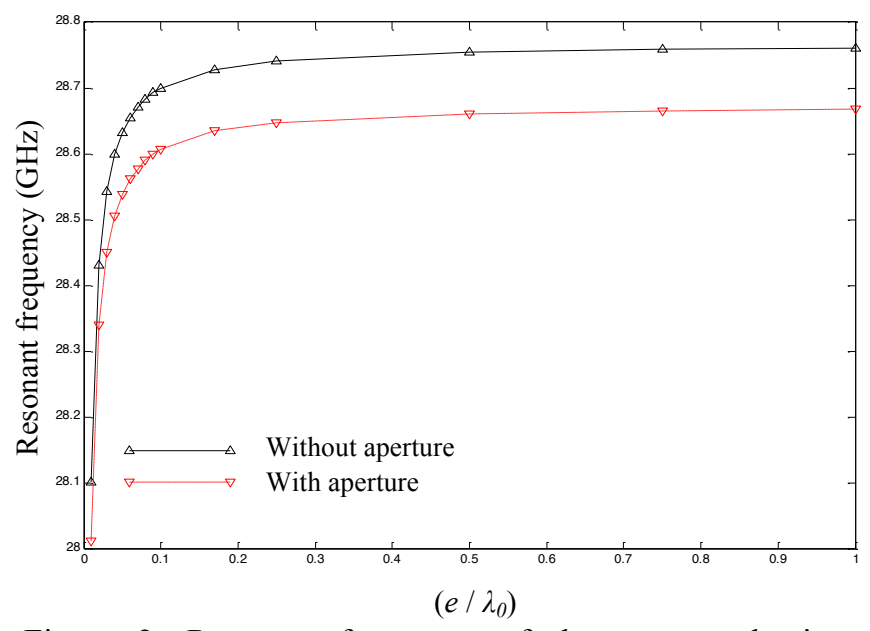

Figure 2: Resonant frequency of the superconducting microstrip antenna with and without a rectangular aperture in the ground plane against normalized thickness of patch.

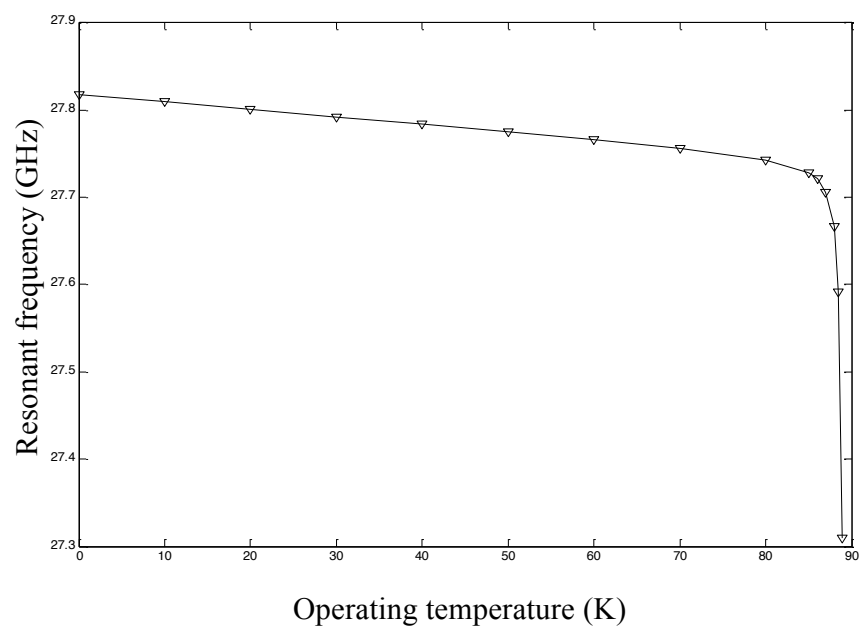

Figure 3: Resonant frequency and bandwidth of the high $T_{c}$ superconducting microstrip patch over ground plane with rectangular aperture against operating temperature; $a=1630 \mu \mathrm{m}, b=935 \mu \mathrm{m}, e=350 \mathrm{~nm}, d=254 \mu \mathrm{m}$, $a_{a} \times b_{a}=163 \mu \mathrm{m} \times 93.5 \mu \mathrm{m}, \sigma_{n}=10^{6} \mathrm{~S} / \mathrm{m}, T_{c}=89 \mathrm{~K}$ and $\lambda_{0}=140 \mathrm{~nm}$.

transition temperature $\sigma_{n}=10^{6} \mathrm{~S} / \mathrm{m}$. The lanthanum aluminate substrate was chosen in the experiment of Richard et al. [7] despite its high permittivity because it allows the growth of high-quality (low surface resistance) YBCO superconducting films [7]. The variation of the permittivity of the lanthanum aluminate substrate with the variation of the temperature, as indicated by the experiment of Richard et al. [7], is taken into account in the present subsection. Figure 3 shows the resonant frequency against the operating temperature. From the results of Figure 3, it can be observed that increasing the temperature will decrease the resonant frequency. This decrease is significant for temperatures near the transition temperature. 
Table 2: Resonant frequencies of superconducting rectangular microstrip patch over ground planes with and without rectangular apertures; $a \times b=1.5 \mathrm{~cm} \times 1 \mathrm{~cm}$,

$$
\varepsilon_{r}=2.32, d=1 \mathrm{~mm}, \sigma_{n}=10^{6} \mathrm{~S} / \mathrm{m}, T_{c}=89 \mathrm{~K}
$$
$\lambda_{0}=140 \mathrm{~nm}$ and $T=50 \mathrm{~K}$.

\begin{tabular}{ccc}
\hline $\begin{array}{c}\text { Aperture size } \\
a_{a} \times b_{a}\end{array}$ & $\begin{array}{c}\text { Resonant } \\
\text { frequency } \\
(\mathrm{GHz})\end{array}$ & $\begin{array}{c}\text { Fractional change } \\
\frac{\Delta f_{r}}{f_{r}}(\%)\end{array}$ \\
\hline 0 & 8.967 & 0 \\
$0.2 a \times 0.2 b$ & 8.725 & 2.70 \\
$0.3 a \times 0.3 b$ & 8.299 & 7.45 \\
$0.4 a \times 0.4 b$ & 7.750 & 13.57 \\
$0.5 a \times 0.5 b$ & 7.171 & 20.03 \\
\hline
\end{tabular}

\subsection{Influence of the aperture cut into the ground plane}

Table 2 presents numerical results for the resonant frequencies of superconducting rectangular microstrip patch over ground planes with and without rectangular apertures. The change in resonant frequency relative to the case without aperture is calculated using the following expression:

$$
\frac{\Delta f_{r}}{f_{r}}=\left|1-\frac{f_{r a}}{f_{r w}}\right|,
$$

where $f_{r a}$ and $f_{r w}$ are respectively, the resonant frequencies of the antenna for with and without aperture cases. It is found that the resonant frequency of the superconducting patch over ground plane without aperture is larger than those obtained with apertures. Note that the influence of the aperture on the resonant frequency increase with the increase of the size of the aperture. The maximum value of the fractional change $(20.03 \%)$ is obtained in the case where $a_{a} \times b_{a}=0.5 a \times 0.5 b$.

\section{Conclusions}

Galerkin's method in the vector Fourier transform domain has been used for the numerical calculation of the resonant frequencies of the $\mathrm{TM}_{01}$ mode of a high $T_{\mathrm{c}}$ superconducting rectangular microstrip antenna with a rectangular aperture cut into the ground plane. The unknowns of the problem are the surface current density on the rectangular patch and the transverse electric field at the plane of the aperture. The complex surface impedance of the superconducting patch has been determined by using London's equation and the two-fluid model of Gorter and Casimir. In order to test the validity of the analysis, numerical results obtained for the resonant frequencies have been compared with previously published data, and good agreement has been found. Numerical results show that the resonant frequencies of the superconducting patches over ground planes without apertures are larger than those obtained with apertures. This result agrees with that discovered theoretically for perfectly conducting patches [3]. Other results also indicate that as the thickness of the superconducting film grows, the resonant frequency increases for antennas without apertures as well as for antennas with rectangular apertures. The increase is more pronounced for thin superconducting films.

\section{References}

[1] S. Benkouda, T. Fortaki, Resonance of rectangular microstrip patch over ground plane with rectangular aperture in the presence of high-permittivity dielectric layer below the aperture, Proc. PIERS11, Marrakech, Morocco, pp. 239-241, 2011.

[2] V. Losada, R.R. Boix, M. Horno, Resonance modes of circular microstrip patches over ground planes with circular apertures in multilayered substrates containing anisotropic and ferrite materials, IEEE Trans. Microwave Theory Tech. 48: 1756-1762, 2000.

[3] T. Fortaki, A. Benghalia, Rigorous Full-wave analysis of rectangular microstrip patches over ground planes with rectangular apertures in multilayered substrates that contain isotropic and uniaxial anisotropic materials, Microwave Opt. Technol. Lett. 41: 496-500, 2004.

[4] S.M. El-Ghazaly, R.B. Hammond, T. Itoh, Analysis of superconducting microwave structures: application to microstrip lines, IEEE Trans. Microwave Theory Tech. 40: 499-508, 1992

[5] D.C. Chung, Perfect impedance matching point of highTc superconducting microstrip antennas, Physica $C$ 354: 95-99, 2001

[6] T. Uwaro, T. Itoh, Numerical techniques for microwave and millimeter-wave passive structures, Wiley, New York, pp.334-380, 1989.

[7] M.A. Richard, K.B. Bhasin, P.C. Claspy, Superconducting microstrip antennas: an experimental comparison of two feeding methods, IEEE Trans. Antennas Propagat. 41: 967-974, 1993.

[8] S.C. Silva, A.G. d'Assuncao, J.R.S. Oliveira, Analysis of high $T_{c}$ superconducting microstrip antenna and arrays, Proc. SBMO/IEEE MTT Symposium, pp. 243-246, 1999. 